\title{
Prevalence and determinants of resistant hypertension among hypertensive patients attending a cardiology clinic in China: a prospective cross-sectional study
}

\author{
Guofan Chen, Fen Chen, Kefeng Sun, Ting Ting Yuan and Xingwei Zhang* \\ Department of Cardiology, The Affiliated Hospital of Hangzhou Normal University, Hangzhou 310015, Hangzhou, China
}

*For correspondence: Email: xingweizhang186@hotmail.com; Tel: 0086-571-88301701

Received: 9 January 2016

Revised accepted: 11 September 2016

\begin{abstract}
Purpose: To determine occurrence and determinants of resistant hypertension (RHT) among patients attending cardiology clinic of the affiliated hospital of Hangzhou Normal University, China.

Methods: An observational prospective cross-sectional study was conducted among patients with hypertension attending the cardiology clinic over a period of 6 months. After identification of patients with $R H T$, various independent co-variants were tested by logistic regression in order to evaluate the determinants of RHT.

Results: Out of 556 patients, 104 (18.7\%) patients had RHT while 67 (12.1\%) patients had uncontrolled blood pressure (BP) in spite of treatment with three antihypertensive drugs including a diuretic; 37 (6.6\%) patients had controlled BP with > three drugs. Obesity (OR: 2.7, $p=0.002$ ], duration of hypertension (OR: 1.8, $p=0.015$ ], presence of diabetes mellitus (OR: $3.6, p<0.001$ ) and ischemic heart disease (OR: 3.2, $p=0.001)$ were significant determinants of resistant hypertension in the study cohort.

Conclusion: The prevalence of RHT found in this study is significantly high, thus indicating a need for greater attention of clinicians to this highly morbid condition. Obese patients and those suffering from diabetes mellitus, ischemic heart disease and chronic diseases should be evaluated for the presence of RHT. Early identification of such patients will provide sufficient time for clinicians to refer patients, as well as modify and/or intensify therapy.
\end{abstract}

Keywords: Resistant hypertension, Risk factors, Hypertension, Stroke, Diabetes mellitus, Ischemic heart disease

Tropical Journal of Pharmaceutical Research is indexed by Science Citation Index (SciSearch), Scopus, International Pharmaceutical Abstract, Chemical Abstracts, Embase, Index Copernicus, EBSCO, African Index Medicus, JournalSeek, Journal Citation Reports/Science Edition, Directory of Open Access Journals (DOAJ), African Journal Online, Bioline International, Open-J-Gate and Pharmacy Abstracts

\section{INTRODUCTION}

Hypertension is a growing problem, affecting about one billion people worldwide; among them two-thirds are from developing countries [1]. Alarmingly, it has been estimated that 1.56 billion adults will have hypertension in 2025 [1]. It is a leading cause of cardiovascular diseases (CVD) and kills 8 million people every year [2]. Hypertension leads to several disabling complications including cardiac failure (CF), retinopathy, stroke, chronic kidney disease (CKD) and atherosclerosis [3]. In order of avoid such complications and associated mortality, a stringent blood pressure (BP) control is mandatory but optimum BP control has been observed only in $1 / 3$ of patients receiving treatment. Such patients with poor BP control fall in the category of resistant hypertension [4]. 
The term "resistant hypertension" refers to uncontrolled blood pressure in spite of the concurrent use of three antihypertensive drugs inclusive of a diuretic, prescribed at optimal dosage. It also includes patients whose blood pressure is controlled but with more than 3 antihypertensive medications [5]. Resistance hypertension is contributed to several factors including old age, alcohol consumption, obesity, sodium intake, adherence and knowledge of therapy and limitation of treatment facilities [6]. Resistant hypertension is usually diagnosed with specialized techniques that are quite expensive and place economic burden on patients and health care systems. However, patients with resistance hypertension bear physical, mental and social disturbances as well. Such patients are more likely to have CVD and end organ damage as compared to patients with controlled blood pressure [7]. Early recognition of hypertension along with appropriate therapy can reduce cardiovascular morbidity and mortality.

Prevalence of hypertension in both developing and developed countries is nearly the same but blood pressure control rates in developing countries are lower than that of developed countries [8]. According to a recent investigation, prevalence of hypertension in China is $26.6 \%$ and blood pressure is observed to be adequately controlled among $11.1 \%$ patients only [9]. A great disparity in blood pressure (BP) control rates $(27.6-46.0 \%)$ among Chinese population has been observed in several studies and it might be contributed to methodological, geographical and population differences in these studies [10-12]. Several efforts have been made to identify possible risk factors of hypertension among Chinese population [10-12] but possible potential determinants of resistant hypertension are unclear in this region. On the other hand, more recently two studies described clinicolaboratory characteristics and risk factors of resistant hypertension among Asian population $[6,13]$. More elaborative research is still needed in order to control resistant hypertension in this region. In this context, we made an attempt to describe the prevalence and determinants of resistance hypertension in patients attending cardiology clinics of Hospital of Hangzhou Normal University.

\section{METHODS}

\section{Patients and study location}

An observational prospective cross-sectional study was conducted among patients attending cardiology follow-up clinics of Hospital of Hangzhou Normal University over a period of 6 months (January 2013 - June 2013). All the patients with hypertension visiting cardiology clinics were identified by using clinic's registers. Patients on regular follow-up were randomly selected for the study purpose. Only those patients who provided informed consent were included in the study. Patients who did not give written informed consent, attended clinic for the first time or less than 18 years of age were excluded from the study. All the patients included in current study were on regular follow-up and most of them (> $90 \%$ ) visited clinic twice a month.

\section{Data collection}

A structured data collection form was used to extract information regarding sociodemographics, baseline illnesses, clinicolaboratory parameters and treatment history. Two of the researchers (FC, KS) also interviewed patients after taking informed consents in order to assess their compliance with treatment, social and family history. On day of visit to the clinic, two blood pressure readings after an interval of ten minutes were recorded in addition to BP readings recorded by attending physician. Patient's previous three readings of blood pressure were also recorded from their file. Patient's height and weight were recorded to calculate BMI $\left(\mathrm{kg} / \mathrm{m}^{2}\right)$.

\section{Definitions}

Hypertension in present study was defined as blood pressure $\geq 140 / 90 \mathrm{mmHg}$ or use of antihypertensive drugs. The term "resistance hypertension" refers to uncontrolled BP in spite use of three antihypertensive drugs (inclusive of at least one diuretic) or patients whose blood pressure is controlled with more than 3 drugs [5]. Chronic Kidney Disease (CKD) was defined as estimated GFR $<60 \mathrm{~mL} / \mathrm{min} / 1.73 \mathrm{~m}^{2}$ where eGFR was calculated by Cockroft-Gault formula. Diagnosis of diabetes mellitus (DM) was based on hyperglycemia recorded by attending clinician or was based on use of hypoglycemic agents. All other co-morbidities i.e. ischemic heart disease and hyperlipidemia were recorded if documented in patient's file. Smokers were referred to patients who were currently smoking at least one cigarette per day. Alcohol consumption was defined as $\geq 1$ alcoholic drink per month. Blood pressure goals or controlled BP in current study were $<140 / 90 \mathrm{mmHg}$ (patients without any comorbidities) or $<130 / 90 \mathrm{mmHg}$ (patients having $\mathrm{DM}$ and renal disease) [14]. BMI values $\geq 27.5$ $\mathrm{kg} / \mathrm{m}^{2}$ were served to define obesity. Salt intake 
was termed as high if daily sodium intake was greater than $3 \mathrm{mg} /$ day (assessed by food history questionnaire).

Medication record of all patients were retrieved from patient's medical file. Drugs taken by all patients were categorized into following categories, Renin angiotensin aldosterone system (RAAS) inhibitors including angiotensin receptor blockers (ARBs) and angiotensin converting enzyme inhibitors (ACEls), beta blockers (BB), alpha blockers $(A B)$, diuretics and calcium channel blockers (CCBs).

\section{Statistical analysis}

A statistical package (SPSS, version 20) was used to analyze the data. Continuous variables were expressed as mean \pm standard deviation (SD) if they were normally distributed while median and range were used if data had skewed distribution. Categorical data were presented as frequency with proportions. Continuous variables were compared between two groups (patients with and without resistant hypertension) using Student t-test while categorical variables were compared using Chi-square test or Fisher's Exact test. Logistic regression was used to determine possible predictors of resistant hypertension. Statistically tested variables and those reported in previous literature were selected to assess their correlation with resistant hypertension. Variables with $p<0.25$ in univariate were considered candidates for multivariate analysis and only significant variables $(p<0.05)$ in multivariate were considered final determinants of resistant hypertension $[15,16]$. Analysis was performed at $95 \%$ confidence interval and $p<0.05$ was considered statistically significant.

\section{Ethical consideration}

This study was approved by the ethical committee of Hospital of Hangzhou Normal University (CASE451/HHNU/2012-A), and the final results as well as the manuscript were also approved by the committee.

\section{RESULTS}

A total of 1089 patients with hypertension attending regular cardiology clinics were selected. Out of the total selected patients, 533 (49\%) patients were excluded from the study; 321 patients refused to participate in the study, 67 patients had very poor compliance with medications, 145 patients were visiting for the first time to the clinic. Finally, 556 (51\%) patients were included in final analysis (Figure 1).

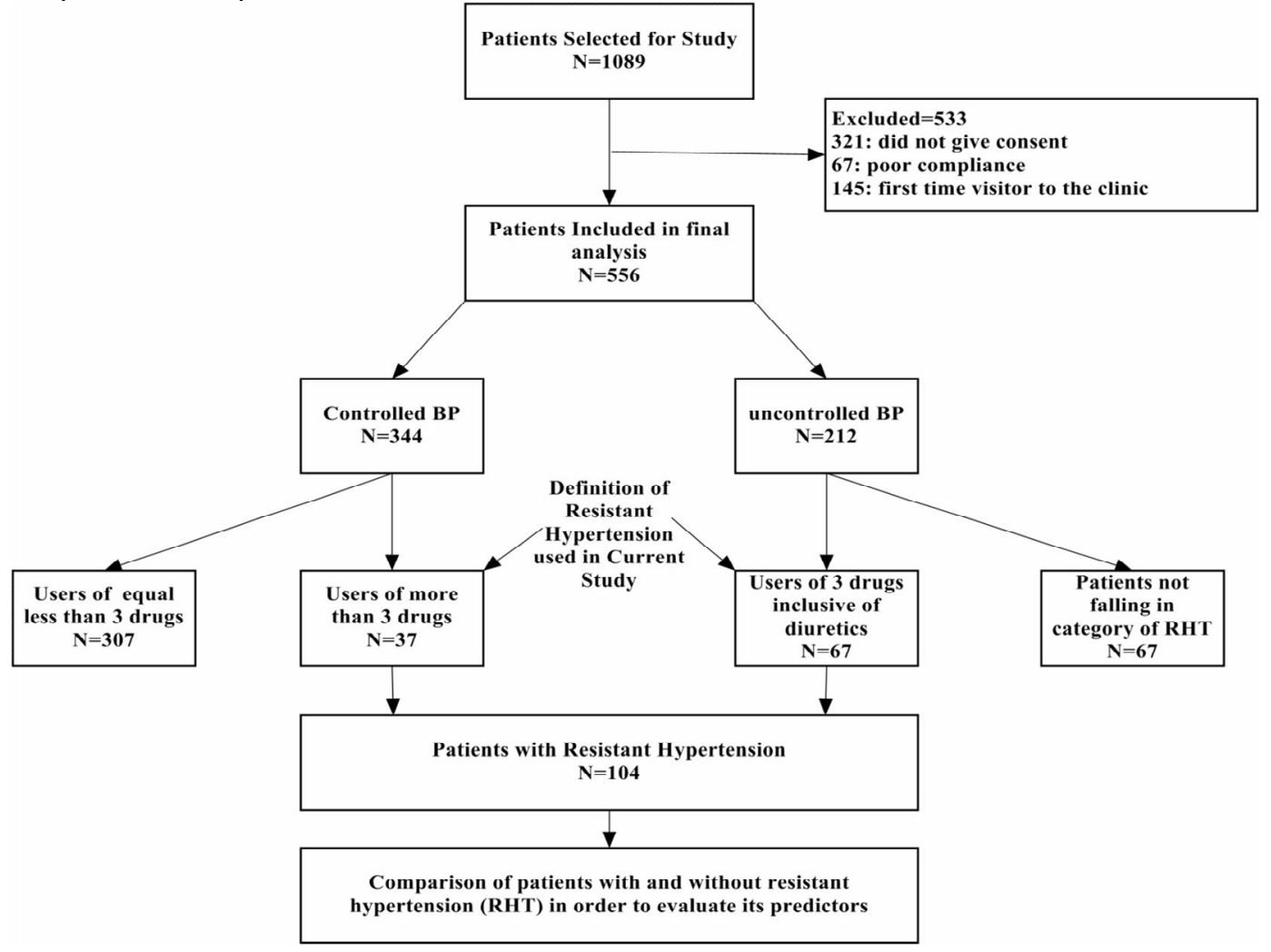

Figure 1: Study methodology flow diagram 
Table 1 describes the socio-demographics of the studied participants. About $60 \%$ of patients had age $>60$ years and gender was about equally distributed among patients (male: $56 \%$ ). The BP readings of all patients during last two visits at least one month apart were recorded and we found both systolic and diastolic blood pressures were high above target range in 212/556 (38.1 $\%)$. Out of total 212 patients with uncontrolled BP, $67(31.6 \%)$ patients were using three antihypertensive medications inclusive of one diuretic. So the proportion of patients with uncontrolled BP among total study participants was $12.1 \%$. On the other hand, 37/556 (6.7\%) patients had controlled hypertension but were using 4 or more medications for their blood pressure control (thirteen patients were on 24 drugs, ten on 5 drugs and three on 6 drugs).

According to the definition of resistant hypertension (RHT) adopted in current study, 104/556 (18.7 \%) patients had resistant hypertension. A total $344 / 556(61.9 \%)$ had controlled blood pressure and among them $111 / 344$ were using three antihypertensive drugs inclusive of a diuretic and 98/344 (28.5\%) patients were using three drugs without diuretics while $37 / 344(10.8 \%)$ patients were using more than 3 antihypertensive drugs to control BP. Demographics, medications and baseline illnesses both patients with and without hypertension were compared in Table 1.
Proportion of obesity was found to be significantly higher $(p=0.014)$ among patients with RHT. Similarly, patients with RHT had prolonged duration of hypertension than patients without RHT $(p=0.045)$. Only dyslipidemia was equally distributed among patients with and without RHT while all others co-morbid conditions were significantly higher among RHT group (Table 1).

The compliance rate among patients with RHT was $100 \%$ while patients without RHT were also compliant (94\%) to their antihypertensive medications. The most commonly prescribed antihypertensive drugs were CCBs $(61.9 \%)$ and ACEls $(55.2 \%)$. The use of ACEls, ARBs, BBs and diuretics was significantly higher among patients with RHT (Table 1). Most commonly prescribed diuretics in current study were furosemide, thiazide and spironolactone. Besides these antihypertensive medications, most commonly prescribed agents were anti-platelets (80\%), lipid lowering agents (39\%) and nitrates (40\%) as seen in this study.

All the statistically tested variables (Table 1) and variables based on previous findings were subjected to logistic regression. Only four predictors in current study were found to be associated with resistant hypertension including obesity, duration of hospitalization and presence of diabetes mellitus and hypertension.

Table 1: Comparison of social demographics, comorbidities and medication use between patients with and without resistant hypertension (RHT)

\begin{tabular}{|c|c|c|c|c|}
\hline Variable & $\begin{array}{l}\text { All patients } \\
\mathrm{N}=556\end{array}$ & $\begin{array}{c}\text { Without RHT } \\
N=452\end{array}$ & $\begin{array}{c}\text { With RHT } \\
\mathrm{N}=104\end{array}$ & $P$-value \\
\hline Age (years, mean $\pm S D$ ) & $64.2 \pm 7.8$ & $63.6 \pm 5.5$ & $65.7 \pm 11.4$ & 0.762 \\
\hline Male, n (\%) & $311(56 \%)$ & $256(56.6 \%)$ & $55(52.9 \%)$ & 0.167 \\
\hline Obesity, n (\%) & $341(61.3 \%)$ & $267(59.1 \%)$ & $74(71.2 \%)$ & 0.014 \\
\hline Smokers, n (\%) & $49(8.8 \%)$ & $40(8.9 \%)$ & $9(8.7 \%)$ & 0.692 \\
\hline Alcohol users, $n(\%)$ & $82(14.8 \%)$ & $65(14.4 \%)$ & $17(16.3 \%)$ & 0.159 \\
\hline High salt intake, $\mathrm{n}(\%)$ & $65(11.7 \%)$ & $56(12.4 \%)$ & $9(8.7 \%)$ & 0.421 \\
\hline Family history of hypertension & $229(41.1 \%)$ & $182(42.3 \%)$ & $47(45.2 \%)$ & 0.665 \\
\hline Duration of hypertension & $10.3 \pm 4.4$ & $9.6 \pm 5.7$ & $11.8 \pm 2.6$ & 0.045 \\
\hline \multicolumn{5}{|l|}{ Comorbidity } \\
\hline DM, n (\%) & $266(47.8 \%)$ & 201 (44.5\%) & $65(62.5 \%)$ & 0.003 \\
\hline CKD, n (\%) & $154(27.2 \%)$ & $113(25 \%)$ & $41(39.4 \%)$ & 0.020 \\
\hline Dyslipidemia, n (\%) & $201(36.2 \%)$ & $169(37.4 \%)$ & $32(30.8 \%)$ & 0.072 \\
\hline \multirow{2}{*}{\multicolumn{5}{|c|}{ Antihypertensive medication }} \\
\hline & & & & \\
\hline CCBs, n (\%) & $344(61.9 \%)$ & $273(60.4 \%)$ & $71(68.3 \%)$ & 0.063 \\
\hline ACEIs, $\mathrm{n}(\%)$ & $307(55.2 \%)$ & $236(52.2 \%)$ & $71(68.3 \%)$ & 0.011 \\
\hline ARBs, $n(\%)$ & $178(32 \%)$ & $132(29.2 \%)$ & $46(44.2 \%)$ & 0.038 \\
\hline Beta blockers, n (\%) & $230(41.4 \%)$ & $176(39 \%)$ & $54(51.9 \%)$ & 0.047 \\
\hline Alpha blockers, n (\%) & $23(4.1 \%)$ & $17(3.8 \%)$ & $6(5.8 \%)$ & 0.288 \\
\hline Diuretics, $n(\%)$ & $221(39.7 \%)$ & $181(36.5 \%)$ & $40(62.5 \%)$ & $<0.001$ \\
\hline
\end{tabular}

DM: diabetes mellitus, CKD: chronic kidney disease, IHD: ischemic heart disease, CCBs: calcium channel blockers, ACEls: angiotensin converting enzyme inhibitors, ARBs: angiotensin receptor blockers 
Table 2: Determinants of resistant hypertension (RHT) by logistic regression (Multivariate analysis)

\begin{tabular}{lccc}
\hline Variable & Adjusted Odds ratio & 95\% confidence interval & $\boldsymbol{P}$-value \\
\hline Age $>60$ years & 2.2 & 1.4 to 4.7 & 0.092 \\
Obesity & 2.7 & 1.1 to 7.8 & 0.002 \\
Longer duration of HTN ${ }^{*}$ & 1.8 & 0.9 to 5.2 & 0.015 \\
Smoking & 1.1 & 0.3 to 4.5 & 0.317 \\
Alcohol use & 0.9 & 0.5 to 2.7 & 0.198 \\
High salt intake & 0.7 & 0.2 to 1.8 & 0.564 \\
DM & 3.6 & 1.9 to 8.5 & $<0.001$ \\
CKD & 1.4 & 1.2 to 5.3 & 0.062 \\
IHD & 3.2 & 2.6 to 11.4 & 0.001 \\
\hline
\end{tabular}

All the variables with $p$ value 0.25 in univariate were subjected to multivariate analysis, Only CKD was found to be significant predictor in univariate [OR: 1.9 (0.7 to 2.6), $p$ value: 0.022], *Longer duration of hypertension refer to duration $\geq 9$ years; HTN: hypertension, DM: diabetes mellitus, CKD: chronic kidney disease, IHD: ischemic heart disease

\section{DISCUSSION}

Resistant hypertension (RHT) portends significant burden to the patients and health care system in terms of cardiovascular events, hence it should be appropriately addressed during clinical practice. The prevalence of RHT in present study was $18.7 \%$ which was comparable with findings of Asian study conducted in Sri Lanka by Kumara et al [6]. On the other hand, prevalence in current study was much higher than reported by Chia et al, another Asian study, conducted in Malaysia [13]. Study location might have contributed to difference in the prevalence. Chia et al enrolled patients from primary care setting. Mostly patients with controlled BP and with less severe conditions usually visit primary care facilities for regular checkup while severe and complicated patients prefer to visit secondary care settings $[13,17,18]$.

Ma et al reported very low prevalence $(1.9 \%)$ of RHT among hypertensive cohort HOT-CHINA study enrolling patients from 148 cities of mainland of China [19]. Wide difference of prevalence of RHT by Ma et al [19] than us might be contributed to several factors. Ma et al [19] excluded patients with new onset of heart disease and cerebrovascular disease. Additionally, the proportion of type 2 diabetes (about $25 \%$ ) among patients with RHT in his was very low while $62.5 \%$ patients with RHT and $44.5 \%$ patients without RHT had diabetes mellitus in current study. On the other hand, treatment program used in HOT-CHINA study was strict and aimed to strengthen the blood pressure control that may lead to low prevalence of RHT in study conducted by Ma et al [19]. Previously published studies demonstrate wide disparity in their findings due to difference in population, definition of RHT used and methodology. These findings suggest the need of well-controlled clinical trials in order to determine accurate prevalence of RHT among Chinese population.

Uncontrolled BP was observed in 212/556 (38.1 $\%$ patients in current study and among them $67 / 212(31.6 \%)$ patients were classified as RHT. The rest $(145 / 212,68.4 \%)$ patients had uncontrolled BP but did not fall under the category of RHT usually refer to uncontrolled or poorly controlled hypertension. Such hypertension is associated with several factors including poor compliance and inadequate therapy. In present study, overall uncontrolled HTN was observed in $26.1 \%$ patients that might be contributed to patient's failure to adhere with therapy (patient's factor) or physician`s failure to intensify therapy (therapeutic or clinical inertia). As most of the patients in current study were compliant with their medications so it can be assumed that therapeutic or clinical inertia in present study is about $26 \%$. These findings are also consistent with previous reports [6]. A careful evaluation to differentiate therapeutic inertia from other causes of uncontrolled hypertension is of particular importance in clinical practice so that such patients could receive optimal therapy.

Four determinants of RHT were observed in current study according to multivariate logistic regression. Obese patients were found to be associated with RHT by odds of 2.7 as compared to non-obese patients. It has been reported that obesity is associated with severe hypertension that increases number of treatments and reduces the rate of blood pressure control [5]. Similar findings were observed by Kumara et al [6]. Duration of hypertension was also found to be associated with RHT in present study. Patients with longer duration of HTN ( $\geq 9$ years) were more likely to be associated (OR: 1.8) with RHT and it is also in concordance with previous study [6]. It might be due to the reason that likelihood of more number of medications are usually 
higher among patients with longer duration of hypertension thus increasing the chances to fall under the definition of resistant hypertension. More elaborative studies are required to evaluate impact of duration of hypertension on resistant hypertension.

Several studies have identified co-morbidities as risk factor of resistant hypertension $[4,6,13,18]$. Patients with DM and IHD had more chances of having resistant hypertension in current study cohort. CKD was only found to be associated with RHT in unadjusted analysis which failed to demonstrate any association in adjusted analysis. It might be due to small sample size ( $\mathrm{n}$ $=41$ ) of patients having concurrent RHT and CKD. Hypertensive patients with DM had more likelihood (OR: 3.6) of having RHT than those without DM. Hypertensive diabetes patients have more chances of having diabetic complications which in turn aggravate hypertension disease and cause difficulty in blood pressure control [20].

Patients with both HTN and DM require strict treatment plan to reduce the risks of CVD and diabetic complications i.e. nephropathy. Renal deterioration was not evaluated in current study due to less number of patients with marked renal function disturbances. It might be due to referral of such patients to nephrology care unit while only patients attending cardiology clinic were enrolled in this study. Ischemic heart disease (IHD) was also found to be a significant determinant of RHT in present study and this finding is consistent with recent report [6].

Recent 8th Joint National Committee (JNC) evidence-based guidelines for the management of high blood pressure in adults recommended initiation of therapy with thiazide diuretic, CCBs, ACEls or ARBs, and if blood pressure is not controlled, then dosage of initial medications should be increased or a second medication should be added [4]. In present study, CCBs and ACEls were frequently prescribed followed by beta blockers, ARBs, diuretics and alpha blockers. Most of the clinical trials showed that use of diuretics in management of HTN is an effective strategy to minimize the risks of stroke and cardiac diseases [21]. However diuretics were not commonly prescribed in present study even less than BBs (39.7 \% vs $41.4 \%)$. It has also been proved that BBs are not as good as other antihypertensive medications for the reduction of morbidity and mortality [22]. As the use of diuretics in current study was low and definition of RHT requires inclusion of diuretics in medications being used by the patients, it is possible that true prevalence in present study might be underestimated.

\section{Limitations of the study}

Some limitations observed with the study include the study period (6 months) therefore causalities among patients were not observed. Larger studies with regular follow-up are required in order to determine relationship of RHT with mortality. Being an observational study, decision on which different medications were used, was not ruled out. Additionally, hospitalization among patients solely related to hypertension and its associated complications is difficult to identify with this study design. Furthermore, there may be bias with the clinician with this study design. Stress levels among patients (that might be related to control of $\mathrm{BP}$ )] was not evaluate and severity of premorbid conditions were not evaluated in current study. But besides these limitations, present study contains several strengths including larger sample size. All the patients were interviewed to ensure that they were compliant to their medications and poorly compliant patients were excluded from the study. Thus, it can be assumed that chances of patients with uncontrolled hypertension due to nonadherence are very low in present study.

\section{CONCLUSION}

The findings of this study indicate that the high prevalence of resistant hypertension that underscore the need for physicians to give attention to identifying resistant hypertension much earlier. Patients with obesity, longer duration of hypertension, diabetes mellitus and ischemic heart disease are significantly associated with RHT. Such-high risk patients should be identified in a timely manner so that referral or intensified therapy could be started.

\section{DECLARATIONS}

\section{Conflict of Interest}

No conflict of interest associated with this work.

\section{Contribution of Authors}

The authors declare that this work was done by the authors named in this article and all liabilities pertaining to claims relating to the content of this article will be borne by them. 


\section{REFERENCES}

1. WHO Regional Office for South East Asia Hypertension fact sheet [internet]. Available from: http://www.searo. who.int/linkfiles/non_communicable_diseases_hyperten sion-fs.pdf. Accessed 21 March, 2012.

2. Global Atlas on Cardiovascular Disease Prevention and Control. Mendis S, Puska P, Norrving B editors. World Health Organization (in collaboration with the World Heart Federation and World Stroke Organization), Geneva, 2011.

3. Hajjar I, Kotchen JM, Kotchen TA. Hypertension: trends in prevalence, incidence, and control. Annu Rev Public Health 2006; 27: 465-490.

4. James PA, Oparil S, Carter BL, Cushman WC, DennisonHimmelfarb C, Handler J, Lackland DT, LeFevre ML, MacKenzie TD, Ogedegbe $O$ et al. Evidence-based guideline for the management of high blood pressure in adults: report from the panel members appointed to the Eighth Joint National Committee (JNC 8). J Am Med Assoc 2014; 311 (5): 507-20.

5. Calhoun $D A$, Jones $D$, Textor $S$, Goff $D C$, Murphy $T P$, Toto RD, White A, Cushman WC, White $W$, sica $D$, Ferdinand K. Resistant hypertension: diagnosis, evaluation, and treatment a scientific statement from the American Heart Association Professional Education Committee of the Council for High Blood Pressure Research. Hypertens 2008; 51(6): 1403-19.

6. Kumara WN, Perera $T$, Dissanayake $M$, Ranasinghe $P$, Constantine GR. Prevalence and risk factors for resistant hypertension among hypertensive patients from a developing country. BMC Res Notes 2013; 6(1) :373.

7. Cuspidi C, Macca G, Sampieri L, Michev I, Salerno M, Fusi V, Severgnini B, Meani S, Magrini F, Zanchetti A. High prevalence of cardiac and extracardiac target organ damage in refractory hypertension. J Hypertens 2001; 19(11): 2063-70.

8. Ibrahim MM, Damasceno A. Hypertension in developing countries. Lancet 2012; 380(9841): 611-9.

9. Gao Y, Chen G, Tian H, Lin L, Lu J, Weng J, Jia W, Ji L, Xiao J, Zhou Z, Ran X. Prevalence of hypertension in China: a cross-sectional study. PLoS One. 2013; 8(6): e65938.

10. Hu DY, Liu LS, Yu JM, Yao CH. National survey of blood pressure control rate in Chinese hypertensive outpatients-China STATUS. Zhonghua Xin Xue Guan Bing Za Zhi 2010; 38(3): 230-8.

11. Sun L, Wang J, Yu J. Cluster analysis on the blood pressure control rate and related factors of hypertensive outpatients in China. Zhonghua Xin Xue Guan Bing Za Zhi 2015; 43(1): 62-7.

12. Wang ZW, Wang $X$, Wang $W$, Chen WW, Zhu ML, Hu SS, Lei ZL, Kong LZ, Liu LS. [The current situation of blood pressure control and the influencing factors on hypertensive patients in residential communities of China]. Zhonghua Liu Xing Bing Xue Za Zhi 2012; 33(9): 903-6.

13. Chia YC, Ching SM. Prevalence and predictors of resistant hypertension in a primary care setting: a crosssectional study. BMC Fam Pract 2014; 15(1): 1.

14. Khan $Y H$, Sarriff A, Adnan AS, Khan AH, Mallhi TH. Blood Pressure and Mortality in Hemodialysis Patients: A Systematic Review of an Ongoing Debate. Ther Apher Dial 2016 (DOI: 10.1111/1744-9987.12406).

15. Mallhi TH, Khan AH, Adnan AS, Sarriff A, Khan $Y H$, Jummaat $F$. Incidence, Characteristics and Risk Factors of Acute Kidney Injury among Dengue Patients: A Retrospective Analysis. PloS one 2015; 10(9): e0138465.

16. Mallhi $T H$, Khan $A H$, Adnan AS, Sarriff A, Khan $Y H$, Jummaat $F$. Clinico-laboratory spectrum of dengue viral infection and risk factors associated with dengue hemorrhagic fever: a retrospective study. BMC Infect Dis 2015; 15(1): 1.

17. Kaplan NM: Resistant hypertension. J Hypertens 2005; 23(8): 1441-1444.

18. Cushman WC, Ford CE, Cutler JA, Margolis KL, Davis $B R$, Grimm RH, Black HR, Hamilton BP, Holland J, Nwachuku $C$ et al. ALLHAT Collaborative Research Group: success and predictors of blood pressure control in diverse North American settings: the Antihypertensive and Lipid-Lowering Treatment to Prevent Heart Attack Trial (ALLHAT). J Clin Hypertens 2002; 4(6): 393-404.

19. Ma W, Zhang $Y$. Low rate of resistant hypertension in Chinese patients with hypertension: an analysis of the HOT-CHINA study. J Hypertens 2013; 31(12): 2386.

20. D'Agostino RB, Vasan RS, Pencina MJ, Wolf PA, Cobain M, Massaro JM, Kannel WB. General cardiovascular risk profile for use in primary care the Framingham Heart Study. Circ 2008; 117(6): 743-53.

21. Wright JT, Dunn JK, Cutler JA, Davis BR, Cushman WC, Ford CE, Haywood LJ, Leenen FH, Margolis KL, Papademetriou V, Probstfield JL. Outcomes in hypertensive black and nonblack patients treated with chlorthalidone, amlodipine, and lisinopril. J Am Med Assoc 2005; 293(13): 1595-608.

22. Wiysonge CS, Bradley HA, Mayosi BM, Maroney RT, Mbewu A, Opie L, Volmink J. Beta-blockers for hypertension. Cochrane Libr 2007. 